\title{
Classical Limit of Demagnetization in a Field Gradient
}

\author{
Leon Heller \\ Biophysics Group, P-21, Los Alamos National Laboratory, \\ Los Alamos, New Mexico, 87544
}

\begin{abstract}
We calculate the rate of decrease of the expectation value of the transverse component of spin for spin- $1 / 2$ particles in a magnetic field with a spatial gradient, to determine the conditions under which a previous classical description is valid. A density matrix treatment is required for two reasons. The first arises because the particles initially are not in a pure state due to thermal motion. The second reason is that each particle interacts with the magnetic field and the other particles, with the latter taken to be via a 2-body central force. The equations for the 1-body Wigner distribution functions are written in a general manner, and the places where quantum mechanical effects can play a role are identified. One that may not have been considered previously concerns the momentum associated with the magnetic field gradient, which is proportional to the time integral of the gradient. Its relative magnitude compared with the important momenta in the problem is a significant parameter, and if their ratio is not small some non-classical effects contribute to the solution.

Assuming the field gradient is sufficiently small, and a number of other inequalities are satisfied involving the mean wavelength, range of the force, and the mean separation between particles, we solve the integro- partial differential equations for the Wigner functions to second order in the strength of the gradient. When the same reasoning is applied to a different problem with no field gradient, but having instead a gradient to the z-component of polarization, the connection with the diffusion coefficient is established, and we find agreement with the classical result for the rate of decrease of the transverse component of magnetization. The corresponding result for this rate in the absence of collisions is much greater.

An approximate value for the ${ }^{3} \mathrm{He}$ self diffusion coefficient based on a hard core potential is found to be $23.1 \mathrm{~mm}^{2} / \mathrm{sec}$ at room temperature and 7 atmospheres. This falls between the results obtained in an NMR experiment having these values of temperature and pressure, in which the spatial gradient of the field is time dependent, and earlier experiments using other techniques, corrected for temperature and pressure variation.
\end{abstract}

\section{Introduction}

The problem of a system possessing magnetization in the presence of a magnetic field having a spatial gradient to its strength was considered classically by Carr and Purcell [1], Torrey [2], and Abragam [3]. The rate of precession of the transverse component of a particle's magnetic moment depends on where it is located in the field, but thermal motion 
and collisions smear this out. This was taken into account in [1] by carrying out a random walk, and in [2] and [3] by adding a diffusion term to the equation for the time rate of change of the magnetization. It leads to a reduction of its transverse component which

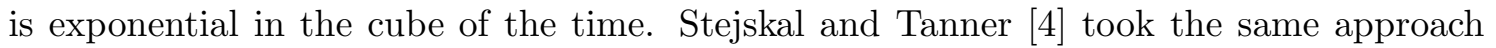
but generalised the problem by permitting the gradient to be time dependent. In the approximate solution obtained there [ [- the reduction of the magnetization has a more complicated time dependence. A classical treatment of NMR experiments involving spatial variation of the magnetization, including the effect of RF pulses, is given in Sodickson and Cory [5]. We shall examine the diminution of transverse magnetization as a quantum mechanics problem to determine under what conditions the classical approach described above is justified.

An isolated spin $1 / 2$ particle is described by a wave function the spin part of which can be written as a linear combination of two basis states, which can be taken to be eigenstates of the $z$-component of spin with eigenvalues $\pm 1 / 2$. Any given values of the relative magnitudes and phases of the coefficients of those two states represents a particle whose spin is pointing in some definite direction in 3-dimensional space. In the presence of a constant magnetic field the spin rotates coherently about the field.

The theoretical description of the process of interest is more complex and proceeds in two steps, precisely following the order in which the experimental conditions are created. Focussing on a single particle, it is located in a heat bath, which means there is a classical probability distribution for its momentum [6]. In addition, the method of preparation (partially polarized) creates a probability distribution for its spin direction [7]. At this first stage already the momentum and spin variables for the particle must be described by a density matrix [6, 7, 8. In the presence of a constant magnetic field the probability distribution of the particle's spin rotates coherently about the field direction, just as for the case of an isolated particle in a pure state. At this stage the central interaction of the particle with the other particles, which are in the same heat bath, has no effect on the spin.

In the second step a gradient to the strength of the field is turned on. The momentum distribution of the particles becomes distorted away from thermal equilibrium because a magnetic moment in the direction of the field experiences a force in the direction of the gradient. Now the 2-body interaction does have an effect. The expectation value of the transverse component of the particle's spin is determined by the off-diagonal elements of the density matrix in spin space, and we shall see that the rate at which those elements decrease with time is affected by the interplay between the two-body interaction and the field gradient.

The effect of (anti)symmetrization on transport properties was studied by Lhuillier and Laloë [9], and included initially in the present work. Those papers were not concerned with the effects of an applied magnetic field; and there appears to be a technical difference between their treatment of the effect of 2-body scattering on the 1-body density matrix and that given in Snider [10], which we follow. This is discussed in the text.

In the Theory section we formulate the problem in terms of the 1-body Wigner distribution functions, and the coupled integro-partial differential equations that they satisfy are obtained as well as the expressions for the expectation value of the particle's spin in 
terms of those functions. Some important parameters are identified, including the momentum associated with the magnetic field gradient. Provided the gradient is sufficiently small and a number of other inequalities are satisfied, the equations are solved in Section 3 by treating the gradient of the magnetic field as a perturbation, to second order in its strength. The successive terms of the solution are gathered together in Section 4, where the result is compared with the classical solution. The approximations encountered along the way are summarized in Section 5 .

A word about terminology. The bulk of the paper is concerned with the quantum mechanical calculation of the expectation value of the spin of a particle. The classical calculation with which it is compared speaks about magnetization, which is the magnetic moment per unit volume. The two quantities are simply related: multiplying the expectation value of the spin by the magnetic moment of a particle and by the number of particles per unit volume gives the magnetization since all the particles are identical.

\section{Theory}

The Hamiltonian for the system is taken to be a sum of 1-body terms, $H^{(1)}$, plus a sum of 2-body potentials $V . H^{(1)}$ contains the kinetic energy plus the interaction energy of the particle's magnetic moment with the applied magnetic field, $B_{z}$, taken to be in the $z$-direction. For spin-1/2 particles this becomes

$$
H^{(1)}=\frac{p^{2}}{2 M}-\frac{\gamma}{2} \hbar \sigma_{z} B_{z}
$$

where $M$ is the mass of the particles, $\gamma$ is the gyromagnetic ratio and $\sigma_{z}$ is the Pauli spin matrix. With the gradient of the magnetic field strength in the direction of the unit vector $\mathbf{u}, B_{z}$ can be written

$$
B_{z}=B_{0}+\mathbf{u} \cdot \mathbf{x} G(t) .
$$

$B_{0}$ is the constant part of the field and $G(t)$ represents the magnitude of the gradient of the field.

We follow the development in Snider [10], but with the inclusion of (anti)symmetrization. There the density matrix for an $\mathrm{N}$ particle system is reduced by taking successive traces down to the 1-body density matrix, $\rho$. It satisfies the equation

$$
\frac{\partial \rho(1)}{\partial t}=-\frac{i}{\hbar}\left(\left[H^{(1)}(1), \rho(1)\right]+\underset{(2)}{\operatorname{Tr}}\left[V(1,2), \rho^{(2)}(1,2)\right]\right)
$$

where the arguments of the operators refer to particles number 1 and 2 and $\rho^{(2)}$ is the 2-body density matrix. (A superscript (1) is omitted on the 1-body $\rho$.) It is indicated in the second term within the parenthesis in (3) that the trace is to be taken over the variables of particle 2. The hierarchy of equations is cut off by neglecting the terms in the equation for $\partial \rho^{(2)} / \partial t$ which involve $\rho^{(3)}$, arguing that in a dilute gas when particles 1 and 2 are interacting the interaction with a third particle can be neglected [10]. With $n$ being the number of particles per unit volume and $a$ the range of the two-body potential, 
this requires that $a \ll d=n^{-1 / 3}$. Normalising to the conditions found in the experiment reported by Schmidt et al. [12] involving a partially polarized gas of ${ }^{3} \mathrm{He}$ atoms, which was performed at room temperature and a pressure of 7 atmospheres,

$$
\frac{a}{d}=0.13\left(\frac{P}{7 a t m} \frac{293^{\circ}}{T}\right)^{1 / 3}\left(\frac{a}{2.4 \cdot 10^{-10} m}\right)
$$

Although the ratio $a / d$ may be on the borderline of being sufficiently small for that particular experiment [12], we shall neglect the possibility of triple collisions, and will continue to take $a=2.4 \cdot 10^{-10} \mathrm{~m}$ as an estimate of the range of the He-He potential when examining the size of other relevant parameters.

We assume that prior to a collision between particles 1 and 2 they are uncorrelated, and hence $\rho^{(2)}$ is just the product of the two 1-body density matrices; this corresponds to the Boltzmann property for the classical distribution functions. Using scattering theory the effect of a collision can be obtained as

$$
\rho^{(2)}(1,2)=\Omega^{(+)} \rho(1) \rho(2) \Omega^{(+) \dagger}
$$

where $\Omega^{(+)}$is the wave operator [11, 10]. When this expression for $\rho^{(2)}$ is inserted into (3) it becomes an equation for the 1-body density matrix $\rho$ alone. We shall seek a solution of this equation to second order in the strength of the gradient $G(t)$. In Lhuillier and Laloë [9] instead of the wave matrix $\Omega^{(+)}$in Eq.(5) the $S$-matrix is used. This relates the density matrix in the distant past, before the collision, to its value in the distant future after the collision is completed. But we are interested in the time development of $\rho$ during a collision [10] and choose the wave matrix, therefore. Our numerical results would be different had we chosen the $S$-matrix.

In the experiment reported by Schmidt et al. [12], a gas of ${ }^{3} \mathrm{He}$ atoms is partially polarized in the $z$-direction and fairly uniform spatially. At $t=0$ it is tipped through an angle $\theta$ thereby producing a component of spin in the plane transverse to the direction of the magnetic field as well as a component in the $z$-direction. It is the diminution of the transverse component with time that was studied, and which we wish to evaluate. The most general form of the density matrix for spin- $1 / 2$ particles can be written

$$
\begin{aligned}
\rho & =\rho_{I} I+\boldsymbol{\rho} \cdot \boldsymbol{\sigma} \\
& =\rho_{I} I+\rho_{z} \sigma_{z}+\frac{1}{2}\left(\rho_{+} \sigma_{-}+\rho_{-} \sigma_{+}\right)
\end{aligned}
$$

where for any operator, $A_{ \pm}=A_{x} \pm i A_{y}$, and the $\sigma_{i}$ are the Pauli spin matrices. The four operators $\rho_{I}, \rho_{z}, \rho_{+}$, and $\rho_{-}=\rho_{+}^{\dagger}$ operate only in ordinary (position or momentum) space.

It is convenient to work with the Wigner distribution function [6] generalized to a $2 \times 2$ matrix to include the spin degree of freedom. This generalization was also made in reference [9]. It is defined by

$$
\begin{aligned}
f_{m m^{\prime}}(\mathbf{x}, \mathbf{p}, t) & =\frac{1}{(\hbar \pi)^{3}} \int \frac{d^{3} q}{(2 \pi \hbar)^{3}}<\mathbf{p}-\mathbf{q}, m|\rho| \mathbf{p}+\mathbf{q}, m^{\prime}>\exp (-2 i \mathbf{x} \cdot \mathbf{q} / \hbar) \\
& =\frac{1}{(\hbar \pi)^{3}} \int d^{3} y<\mathbf{x}-\mathbf{y}, m|\rho| \mathbf{x}+\mathbf{y}, m^{\prime}>\exp (2 i \mathbf{p} \cdot \mathbf{y} / \hbar)
\end{aligned}
$$


where the indices $m$ and $m^{\prime}$ take the values $\pm 1 / 2$. It follows from the fact that $\rho$ is hermitean that the $2 \times 2$ matrix $f$ is also. We also need the inverse relation

$$
<\mathbf{p}, m|\rho| \mathbf{p}^{\prime}, m^{\prime}>=(2 \pi \hbar)^{3} \int d^{3} x f_{m m^{\prime}}\left(\mathbf{x},\left(\mathbf{p}+\mathbf{p}^{\prime}\right) / 2, t\right) \exp \left[i\left(\mathbf{p}^{\prime}-\mathbf{p}\right) \cdot \mathbf{x} / \hbar\right] .
$$

Taking the partial derivative of Eq.(7) or (8) with respect to time and using Eq.(3) gives

$$
\frac{\partial f_{m m^{\prime}}}{\partial t}=S_{m m^{\prime}}^{K}+S_{m m^{\prime}}^{\gamma}+S_{m m^{\prime}}^{V}
$$

where $S^{K}$ arises from the kinetic energy term in $H^{(1)}, S^{\gamma}$ from the magnetic energy term in $H^{(1)}$, and $S^{V}$ from the 2-body potential energy term. $S^{K}$ is the well known flow term

$$
S_{m m^{\prime}}^{K}=-\frac{1}{M} \mathbf{p} \cdot \nabla f_{m m^{\prime}}
$$

To obtain $S^{\gamma}$ requires evaluation of the commutator $\left[\sigma_{z} B_{z}, \rho\right]$. Making use of Eqs. (2) and (6) yields

$$
\begin{aligned}
S_{1 / 2}^{\gamma} & =-\frac{\gamma}{2} \hbar G(t) \mathbf{u} \cdot \nabla_{p} f_{1 / 2} \\
S_{-1 / 2}^{\gamma} & =\frac{\gamma}{2} \hbar G(t) \mathbf{u} \cdot \nabla_{p} f_{-1 / 2} \\
S_{+}^{\gamma} & =-i \gamma B_{z} f_{+}
\end{aligned}
$$

where we have introduced abbreviated notations for all the $S$ terms as follows: $S_{1 / 2} \equiv$ $S_{1 / 21 / 2} ; S_{-1 / 2} \equiv S_{-1 / 2-1 / 2} ;$ and $S_{+} \equiv S_{-1 / 2} 1 / 2 ;$ and the same definitions apply to the components of the Wigner distribution functions $f$. We shall be particularly interested in the function $f_{+}$, which involves the off-diagonal elements of $\rho$ in the 2-dimensional spin space. In conjunction with Eq.(10) for the time rate of change of the distribution functions, the interpretation of Eqs. (12) and (13) is as follows. A magnetic moment having a component in the direction of (or opposite to) a magnetic field whose strength has a spatial gradient will experience a force in the direction of (or opposite to) that gradient. Eq.(14) says that a magnetic moment transverse to the direction of the magnetic field undergoes Larmor precession about the field.

With the density matrix normalized to $\operatorname{Tr}(\rho)=N$, the total number of particles, it is straightforward to obtain the following results for $n$, the number of particles per unit volume, and $\langle\boldsymbol{\sigma}\rangle$, the expectation value of the particle's spin at position $\mathbf{x}$ and time $\mathrm{t}$

$$
n=\int d^{3} p\left[f_{1 / 2}(\mathbf{x}, \mathbf{p})+f_{-1 / 2}(\mathbf{x}, \mathbf{p})\right]
$$

and

$$
\begin{aligned}
<\sigma_{z}> & =\frac{\int d^{3} p\left[f_{1 / 2}(\mathbf{x}, \mathbf{p})-f_{-1 / 2}(\mathbf{x}, \mathbf{p})\right]}{\int d^{3} p\left[f_{1 / 2}(\mathbf{x}, \mathbf{p})+f_{-1 / 2}(\mathbf{x}, \mathbf{p})\right]} \\
<\sigma_{+}> & =\frac{2 \int d^{3} p f_{+}(\mathbf{x}, \mathbf{p}, t)}{\int d^{3} p\left[f_{1 / 2}(\mathbf{x}, \mathbf{p})+f_{-1 / 2}(\mathbf{x}, \mathbf{p})\right]}
\end{aligned}
$$


Once these quantities have been obtained the net magnetic moment per unit volume, $\mathbf{M}$, can be obtained simply by multiplying $\langle\boldsymbol{\sigma}\rangle$ by the magnetic moment of a single particle, $\gamma \hbar / 2$, and the number of particles per unit volume, $n$.

We now turn to $S^{V}$, the collision term in Eq.10). Introducing the spin and momentum variables for particle 2, over which the trace indicated in Eq.(3) will be taken, and the antisymmetrization operator $\left(I+\epsilon P_{12}\right)$ where $P_{12}$ permutes all the variables of particles 1 and 2 and $\epsilon=-1$ for fermions, $S^{V}$ can be written

$$
\begin{aligned}
S_{m m^{\prime}}^{V} & (\mathbf{x}, \mathbf{p})=-\frac{i}{\hbar} \frac{1}{(\hbar \pi)^{3}} \sum_{m_{2}} \int \frac{d^{3} q}{(2 \pi \hbar)^{3}} \frac{d^{3} p_{2}}{(2 \pi \hbar)^{3}} \\
\cdot & <\mathbf{p}-\mathbf{q}, m ; \mathbf{p}_{\mathbf{2}}, m_{2}\left|\left(I+\epsilon P_{12}\right)\left[V, \rho^{(2)}(1,2)\right]\right| \mathbf{p}+\mathbf{q}, m^{\prime} ; \mathbf{p}_{2}, m_{2}> \\
\quad & \exp (-2 i \mathbf{x} \cdot \mathbf{q} / \hbar) .
\end{aligned}
$$

It is sufficient to antisymmetrize just one of the states because $V$ and $\rho^{(2)}$ are symmetric under the interchange of the two particles. In the Appendix we will develop the collision term making two approximations. The first is to assume that the 2-particle potential energy $V$ consists purely of a central interaction. For example, at a separation of $2.4 \AA$ the moment-moment interaction energy of two ${ }^{3} \mathrm{He}$ atoms is $\approx 10^{-8} \mathrm{mev}$, whereas the central interaction is $\approx 1 \mathrm{mev}$. The second approximation amounts to assuming that the gradient of the magnetic field strength is sufficiently small; this comes about as follows.

Examination of Eqs.(10) through (14), which represent the contributions to the time derivative of the respective distribution functions, shows that only $S_{+}^{\gamma}$ contains dependence on position via the gradient term in the magnetic field, $B_{z}$. Neglecting the collision terms $S^{V}$ for the moment, would lead to the following conclusions. With the particle density $n$ and the expectation value of the spin initially independent of position, $n$ and $\left\langle\sigma_{z}\right\rangle$ remain so since $f_{ \pm 1 / 2}$ are independent of position. $n$ is, of course, also independent of time, and $\left\langle\sigma_{z}\right\rangle$ as well since $\sigma_{z}$ commutes with the Hamiltonian. On the other hand $f_{+}$ would contain a factor $\exp \left[-i \gamma\left(B_{0} t+\mathbf{u} \cdot \mathbf{x} F(t)\right)\right]$ as the only position dependence in the problem, where

$$
F(t)=\int_{0}^{t} d t^{\prime} G\left(t^{\prime}\right)
$$

We show below that all these conclusions remain valid with collisions and write, therefore,

$$
f_{+}(\mathbf{x}, \mathbf{p}, t)=g_{+}(\mathbf{p}) \exp \left[-i \gamma\left(B_{0} t+\mathbf{u} \cdot \mathbf{x} F(t)\right)\right] .
$$

The product $\gamma F(t)$ is the wave number associated with the magnetic field gradient [5]; in that reference it is designated as $k$.

The consequences for the matrix elements of $\rho$ can be seen as follows. From Eq.(9) those matrix elements with $m=m^{\prime}$, since they arise from $f_{ \pm 1 / 2}$ which do not depend on position, are local in momentum space,

$$
<\mathbf{p}, m|\rho| \mathbf{p}^{\prime}, m>=(2 \pi \hbar)^{3} f_{m}(\mathbf{p})(2 \pi \hbar)^{3} \delta^{(3)}\left(\mathbf{p}^{\prime}-\mathbf{p}\right) .
$$

On the other hand, for the off-diagonal matrix elements we have

$$
\begin{gathered}
<\mathbf{p},-1 / 2|\rho| \mathbf{p}^{\prime}, 1 / 2>=(2 \pi \hbar)^{3} g_{+}\left(\left(\mathbf{p}+\mathbf{p}^{\prime}\right) / 2\right) \exp \left(-i \gamma B_{0} t\right) \\
\cdot(2 \pi \hbar)^{3} \delta^{(3)}\left(\mathbf{p}^{\prime}-\mathbf{p}-\gamma \hbar \mathbf{u} F(t)\right) .
\end{gathered}
$$


Position dependence of a distribution function is sometimes neglected in the collision term on the assumption that the quantity that is varying (the strength of the magnetic field in the present case) changes little over the distance of a mean free path [13]. But the $\delta$-function in Eq.(22) contains another relevant quantity, $\gamma \hbar \mathbf{u} F(t)$, that has nothing to do with the mean free path. It is a momentum that arises from the oppositely directed forces felt by particles with their moments in the direction of the field, or opposite to it, due to the field gradient. After inserting two complete sets of 2-body spin and momentum states into Eq.(18) matrix elements of the type $<\mathbf{p},-1 / 2|\rho| \mathbf{p}^{\prime}, 1 / 2>$ appear linearly in the collision term $S_{+}^{V}$ and bilinearly (or not at all) in $S_{ \pm 1 / 2}^{V}$. When the $\delta$-function from Eq.(22) is combined with the two momentum conserving $\delta$-functions from the $t$ - and $\Omega^{(+)}$-matrix elements [see (A-1) and (A-2)] it has the following effects. In Eq.(18) for $S_{ \pm 1 / 2}^{V}$ the integration variable $\mathbf{q}$ is required to be zero; but in $S_{+}^{V}$ it becomes $\gamma \hbar F(t) \mathbf{u} / 2$. Consequently $S_{+}^{V}$ acquires a factor $\exp \left[-i \gamma\left(B_{0} t+\mathbf{u} \cdot \mathbf{x} F(t)\right)\right]$, and as a result every term in Eq.(10) for $\partial f_{+} / \partial t$ has that factor. This confirms the statements above that $f_{ \pm 1 / 2}$ are independent of position and $f_{+}$has the structure shown in Eq.(20). No assumption about the magnitude of $F(t)$ has been made at this point.

If the magnitude of $\gamma \hbar F(t)$ were not sufficiently small compared to the important momenta in the problem there would be significant quantum mechanical consequences for the collision terms of the displaced argument of the $\delta$-function in Eq. (22). The arguments of $g_{+}$and the arguments of the $t$-matrix elements would be shifted by amounts of order $\gamma \hbar F(t) \mathbf{u}$. We shall delay discussion of the magnitude of the effect on the collision terms due to the altered $t$-matrix elements, but note here that it would even lead to the presence of non-energy-conserving matrix elements.

The effect of the variation in $g_{+}$can be seen directly. Although the distribution functions are of the Fermi-Dirac type, we shall be interested in conditions of negligible degeneracy, $n \bar{\lambda}^{3} \ll 1$, where $\bar{\lambda}$ is the mean wavelength of the particles. In that case we can use the Maxwell-Boltzmann distribution, and its momentum derivative is of order $\left(p / \bar{p}^{2}\right)$ times the function itself, where $\bar{p}$ is the mean momentum at temperature $T$. The fractional shift in the magnitude of $g_{+}$, therefore, is of order $\gamma \hbar F(t) / \bar{p}$. We shall estimate it by normalising to the experimental conditions found in reference [12]. The gyromagnetic ratio for ${ }^{3} \mathrm{He}$ is $204 \mathrm{MHz} /$ Tesla, and the maximum value of $F(t)$ in that experiment, designated $F_{M}$, is $3.5 \cdot 10^{-7}$ Tesla-sec $/ \mathrm{cm}$. Estimating $\bar{p}$ by the mean value of the momentum at temperature $T$, we obtain

$$
\frac{\gamma \hbar F(t)}{\bar{p}} \approx 9.5 \cdot 10^{-8}\left(\frac{\gamma}{\gamma^{3} \mathrm{He}}\right)\left(\frac{F(t)}{F_{M}}\right)\left(\frac{M_{3} \mathrm{He}}{M} \frac{293^{\circ}}{T}\right)^{1 / 2} .
$$

For any other set of experimental parameters it is only necessary to insert the appropriate values of the gyromagnetic ratio $\gamma$, the temperature $T$, the molecular mass $M$, and the integrated strength of the gradient of the magnetic field, $F(t)$. Note that a larger strength of the field gradient or a smaller temperature would increase the ratio in Eq.(23), but the variation with $T$ is slow.

For the remainder of the paper we shall assume that the shift in the arguments of $g_{+}$and the $t$-matrix elements can be neglected, and this leads to the expressions for the 
collision terms presented in Eqs. $\mathrm{A}-17$ ) and (A-18). Eqs.(10) then become the following set of coupled nonlinear integro- partial differential equations.

$$
\begin{aligned}
& \frac{\partial f_{1 / 2}(\mathbf{p})}{\partial t}=-\frac{\gamma}{2} \hbar G(t) \mathbf{u} \cdot \nabla_{p} f_{1 / 2}(\mathbf{p})+S_{1 / 2}^{V}(\mathbf{p}) \\
& \frac{\partial f_{-1 / 2}(\mathbf{p})}{\partial t}=\frac{\gamma}{2} \hbar G(t) \mathbf{u} \cdot \nabla_{p} f_{-1 / 2}(\mathbf{p})+S_{-1 / 2}^{V}(\mathbf{p})
\end{aligned}
$$

and in the equation for $\partial f_{+} / \partial t$ the derivative of the exponential with respect to time cancels the term $S_{+}^{\gamma}$ leaving

$$
\frac{\partial g_{+}(\mathbf{p})}{\partial t}=i \frac{\gamma}{M} \mathbf{u} \cdot \mathbf{p} F(t) g_{+}(\mathbf{p})+\bar{S}_{+}^{V}(\mathbf{p})
$$

\section{Perturbation Expansion}

Our approach for solving these equations, consistent with the approximation described above, is to treat the strength of the field gradient, $G(t)$, as a perturbation. Since it is multiplied by the gyromagnetic ratio $\gamma$ we shall expand the distribution functions up to second order in $\gamma$,

$$
f_{ \pm 1 / 2}=f_{ \pm 1 / 2}^{(0)}+\gamma f_{ \pm 1 / 2}^{(1)}+\gamma^{2} f_{ \pm 1 / 2}^{(2)}+O\left(\gamma^{3}\right)
$$

and

$$
g_{+}=g_{+}^{(0)}+\gamma g_{+}^{(1)}+\gamma^{2} g_{+}^{(2)}+O\left(\gamma^{3}\right)
$$

This procedure converts the collision terms $S^{V}$ from being bilinear in the functions $f$ and $g$ to being linear in the successive approximations $f^{(i)}$ and $g^{(i)}$.

\section{Zeroth Order}

The terms of order $\gamma^{0}$ in Eqs. (24) - (26) represent the initial condition in which the probability of finding the particle with a given momentum is given by a thermal distribution, and the expectation value of its spin is specified; both quantities are initially independent of position. Those distribution functions can be written

$$
f_{ \pm 1 / 2}^{(0)}(\mathbf{p})=n_{ \pm 1 / 2} B^{(M)}(p)
$$

and

$$
g_{+}^{(0)}(\mathbf{p})=n_{+} B^{(M)}(p)
$$

where

$$
B^{(M)}(p)=\left(\beta^{(M)} / \pi\right)^{3 / 2} \exp \left(-\beta^{(M)} p^{2}\right)
$$

is the the Maxwell-Boltzmann distribution for a particle of mass $M$, and $\beta^{(M)}=(1 / 2 M k T)$. As expected from conservation of energy [13], when these zeroth order distribution functions are inserted in the collision integrals they can be shown to vanish, confirming that 
Eqs. (24) - (26) are satisfied to this order. The unitarity relation gets used here and in the subsequent development [see Appendix]. The significance of the normalization constants can be seen from Eqs.(15) to (17), since they determine the initial values of the number density, $n$, and the expectation value of the spin, $\left\langle\boldsymbol{\sigma}>\right.$ : $n=n_{1 / 2}+n_{-1 / 2},\left\langle\sigma_{z}\right\rangle=$ $\left(n_{1 / 2}-n_{-1 / 2}\right) / n$, and $<\sigma_{+}>=n_{+} / n$.

\section{First Order}

At the next order in $\gamma$ the distribution functions are distorted in the direction $\mathbf{u}$ of the gradient, so we write

$$
f_{ \pm 1 / 2}^{(1)}(\mathbf{p})=n_{ \pm 1 / 2} B^{(M)}(p) \mathbf{u} \cdot \mathbf{p} h_{ \pm 1 / 2}^{(1)}
$$

and

$$
g_{+}^{(1)}(\mathbf{p})=n_{+} B^{(M)}(p) \mathbf{u} \cdot \mathbf{p} h_{+}^{(1)} .
$$

In principle the functions $h^{(1)}$ could depend on the magnitude of the momentum, and a systematic expansion in a complete set of Sonine polynomials, for example [13], could be undertaken. We shall take the $h^{(1)}$ functions to be independent of $p$, thereby limiting that expansion to just the lowest term. Note that these first order terms in the distribution functions contribute nothing to the number density and the expectation value of the spin, Eqs. (15) - (17), because their integrals over all momenta vanish. As expected if $\left\langle\sigma_{z}\right\rangle \neq 0$ the particles acquire a non-zero expectation value of momentum, and it can be shown to be proportional to the product of $\left(n_{1 / 2}-n_{-1 / 2}\right) / n$ and $\gamma \hbar \mathbf{u} F(t)$. The latter factor is the same one we required to be small compared to thermal momenta in the discussion above.

Now pick out of Eqs. (24) - (26) all the terms of first order in $\gamma$. Since the functions $h^{(1)}$ depend only on the time, these become coupled ordinary first order linear differential equations

$$
\begin{aligned}
& n_{1 / 2} B^{(M)}(p) \mathbf{u} \cdot \mathbf{p} \frac{d h_{1 / 2}^{(1)}}{d t}=n_{1 / 2} \hbar \beta^{(M)} B^{(M)}(p) \mathbf{u} \cdot \mathbf{p} G(t)+S_{1 / 2}^{V(1)} \\
& n_{-1 / 2} B^{(M)}(p) \mathbf{u} \cdot \mathbf{p} \frac{d h_{-1 / 2}^{(1)}}{d t}=-n_{-1 / 2} \hbar \beta^{(M)} B^{(M)}(p) \mathbf{u} \cdot \mathbf{p} G(t)+S_{-1 / 2}^{V(1)}
\end{aligned}
$$

and

$$
n_{+} B^{(M)}(p) \mathbf{u} \cdot \mathbf{p} \frac{d h_{+}^{(1)}}{d t}=i \frac{1}{M} n_{+} B^{(M)}(p) \mathbf{u} \cdot \mathbf{p} F(t)+\bar{S}_{+}^{V(1)}
$$

The expressions for the first order collision terms $S^{V(1)}$ are given in the Appendix, where the above equations are developed into Eqs. (A-24) to (A-26).

There are three quantities in those equations, $I_{U}, I_{I}$, and $I_{\pi}$ that depend upon the details of the 2-body potential energy; see Eq. A-23). $I_{U}$, which is the average over a Maxwell-Boltzmann distribution of the cube of the momentum multiplied by the transport cross section, enters into the direct terms. $I_{I}$ and $I_{\pi}$, which involve the $t$-matrix elements 
themselves and not just their absolute square, appear only in the exchange terms, i.e., those proportional to $\epsilon$, which arise from (anti)symmetrization of the wave functions. To study the relative importance of the latter it is necessary to specify the 2-body potential energy function for the problem of interest and calculate the $t$-matrix that it produces. For He the interatomic potential becomes strongly repulsive at distances less than $2.4 \AA$ [14], so we shall use a hard sphere potential as a guide; and there are two limiting cases for which analytic approximations to the scattering amplitude can be obtained: $k a / \hbar \gg 1$ and $k a / \hbar \ll 1$ where $\mathrm{k}$ is the relative momentum of the colliding particles and $a$ is the radius of the hard sphere potential. Numerical calculations of the quantum mechanical scattering amplitude in the short wavelength limit [15] are in qualitative and even semiquantitative agreement with the classical scattering of a scalar wave on a rigid sphere, as presented in Morse and Feshbach [16], and also by the scattering of an electromagnetic wave by a perfectly conducting sphere, given in Jackson 17]. That $t$-matrix takes the approximate form

$$
t(k, \theta) \approx \frac{2 \pi \hbar^{2}}{\mu} \frac{a}{2}\left(i \frac{k a}{\hbar} \frac{(1+\cos \theta) J_{1}\left(\frac{k a}{\hbar} \sin \theta\right)}{\frac{k a}{\hbar} \sin \theta}+\exp \left[-2 i \frac{k a}{\hbar} \sin \frac{\theta}{2}\right]\right) \quad(k a / \hbar \gg 1)
$$

where the first term arises from scattering by the shadow side and the second term from the illuminated side; $\mu$ is the reduced mass. The pure imaginary shadow scattering amplitude dominates in the small angle region $\theta \lesssim \hbar / k a$, and oscillates rapidly with decreasing amplitude for larger angles.

Normalizing once again to the conditions in reference 12 gives

$$
\frac{\bar{k} a}{\hbar}=12.5\left(\frac{M}{M_{3} \mathrm{He}} \frac{T}{293^{\circ}}\right)^{1 / 2} \frac{a}{2.4 \AA}
$$

where we have chosen $2.4 \AA$ as the hard sphere radius for a gas of ${ }^{3} \mathrm{He}$ atoms. Although it would require a very low temperature to get into the long wavelength limit, even a moderately low temperature, $\approx 10^{\circ}$, would introduce significant corrections to the $t$-matrix as well as the first order collision terms, $S^{V(1)}$. In particular, contributions to $S^{V(1)}$ arising from antisymmetrization of the wave functions would not be negligible and this would produce important modifications to the remainder of the paper. Keeping that in mind we now estimate the magnitudes of $I_{U}, I_{I}$ and $I_{\pi}$ in Eq. A-23) using the short wavelength expression (37). It should be noted that this limit, $\bar{\lambda} / a \ll 1$, together with the low density assumption, $n a^{3} \ll 1$, imply $n \bar{\lambda}^{3} \ll 1$, which is the negligible degeneracy assumption made earlier. Using Eq.(37) we can also verify the assumption made earlier that the shift in the momentum (or momentum transfer) argument of the $t$-matrix element by the amount $\gamma \hbar F(t)$ has a negligible effect for the conditions of reference [12].

Although the shadow scattering and the scattering from the illuminated side each contribute $\pi a^{2}$ to the total cross section, the factor $(1-\cos \theta)$ in Eq. (A-22) for the transport cross section eliminates the shadow contribution, hence $\sigma_{U}(k)=\pi a^{2}$; and from Eq.(A-23) $I_{U}=\pi \overline{k^{3}} a^{2}$, where $\overline{k^{3}}$ is the average of $k^{3}$ over a Maxwell-Boltzmann distribution for a particle having mass $\mu$. It takes a considerable amount of algebra to show that when the short wavelength expression Eq.(37) for the scattering amplitude is inserted into Eqs. A13), (A-22) and (A-23), $I_{I}$ is smaller than $I_{U}$ by $O\left[(\bar{k} a / \hbar)^{-2}\right] . I_{\pi}$ is even smaller, being 
$O\left(\exp \left[-(\bar{k} a / \hbar)^{-2}\right]\right)$. Therefore all the terms proportional to $\epsilon$ in Eqs. (A-24)-( $\left.\mathrm{A}-26\right)$ can be neglected.

In the short wavelength limit Eqs. $(\mathrm{A}-24)-(\mathrm{A}-26)$ for the time derivative of the first order functions $h^{(1)}$ become

$$
\begin{aligned}
& n_{1 / 2} \frac{d h_{1 / 2}^{(1)}}{d t}=n_{1 / 2} \hbar \beta^{(M)} G(t)+\frac{4 \beta^{(M)}}{3 M} n_{1 / 2} n_{-1 / 2}\left[h_{-1 / 2}^{(1)}-h_{1 / 2}^{(1)}\right] I_{U} \\
& n_{-1 / 2} \frac{d h_{-1 / 2}^{(1)}}{d t}=-n_{-1 / 2} \hbar \beta^{(M)} G(t)-\frac{4 \beta^{(M)}}{3 M} n_{1 / 2} n_{-1 / 2}\left[h_{-1 / 2}^{(1)}-h_{1 / 2}^{(1)}\right] I_{U}
\end{aligned}
$$

and

$$
\frac{d h_{+}^{(1)}}{d t}=i \frac{1}{M} F(t)+\frac{4 \beta^{(M)}}{3 M}\left[n_{1 / 2} h_{1 / 2}^{(1)}+n_{-1 / 2} h_{-1 / 2}^{(1)}-\left(n_{1 / 2}+n_{-1 / 2}\right) h_{+}^{(1)}\right] I_{U} .
$$

Summing the first two equations gives

$$
n_{1 / 2} h_{1 / 2}^{(1)}+n_{-1 / 2} h_{-1 / 2}^{(1)}=\left(n_{1 / 2}-n_{-1 / 2}\right) \hbar \beta^{(M)} F(t)
$$

where $F(t)$ is the time integral of the field gradient, Eq.(19). There is no need to solve for $h_{1 / 2}^{(1)}$ and $h_{-1 / 2}^{(1)}$ separately because only the combination shown in Eq.(42) is needed in the equation for $d h_{+}^{(1)} / d t$; and furthermore the terms of first order in $\gamma$ do not contribute to the magnetization.

The solutions for the real and imaginary parts of $h_{+}^{(1)}$ are

$$
\operatorname{Re}_{+}^{(1)}=\frac{4 \beta^{(M)}}{3 M}\left(n_{1 / 2}-n_{-1 / 2}\right) \hbar \beta^{(M)} I_{U} \exp (-\alpha t) \int_{0}^{t} d t^{\prime} F\left(t^{\prime}\right) \exp \left(\alpha t^{\prime}\right)
$$

and

$$
\operatorname{Im} h_{+}^{(1)}=\frac{1}{M} \exp (-\alpha t) \int_{0}^{t} d t^{\prime} F\left(t^{\prime}\right) \exp \left(\alpha t^{\prime}\right)
$$

where

$$
\alpha=\frac{4 \beta^{(M)}}{3 M} n I_{U} .
$$

$n=n_{1 / 2}+n_{-1 / 2}$ is the total number of particles per unit volume.

$\alpha$ can also be expressed in terms of the diffusion constant, $D$, for a related problem. Suppose there is no magnetic field and no transverse component of the magnetization, but there is a spatial gradient to the $z$-component of magnetization. This reduces to a standard 2-component problem with equal masses (spin up and spin down); and if the diffusion constant is calculated with the same approximation as above, namely keeping only the leading term in an expansion in Sonine polynomials, then 18]

$$
\alpha=\frac{k T}{M D} .
$$


The qualitative significance of $\alpha^{-1}$ can be seen from Eq.(45) or its expression in terms of the diffusion constant: it is the mean free time between collisions. Normalising to the conditions in reference 12 ]

$$
\alpha=3.5 \cdot 10^{10} \mathrm{sec}^{-1}\left(\frac{P}{7 \mathrm{~atm}}\right)\left(\frac{M_{3} \mathrm{He}}{M} \frac{293^{\circ}}{T}\right)^{1 / 2}\left(\frac{a}{2.4 \cdot 10^{-10} \mathrm{~m}}\right)^{2} .
$$

Provided $G(t)$, and therefore $F(t)$, vary on a time scale much longer than $\alpha^{-1}$, the integrals in Eqs.(43) and (44) are well approximated for $t>>\alpha^{-1}$ by

$$
\exp (-\alpha t) \int_{0}^{t} d t^{\prime} F\left(t^{\prime}\right) \exp \left(\alpha t^{\prime}\right) \cong \frac{F(t)}{\alpha}
$$

This leads to

$$
\operatorname{Re}_{+}^{(1)}=\frac{n_{1 / 2}-n_{-1 / 2}}{n} \hbar \beta^{(M)} F(t)
$$

and

$$
\operatorname{Im} h_{+}^{(1)}=\frac{1}{M \alpha} F(t)
$$

For conditions similar to those of reference 12] $\operatorname{Re} h_{+}^{(1)}$ is much smaller than $\operatorname{Im} h_{+}^{(1)}$, their ratio being, at most, that of the mean wavelength to the mean free path; this will be small if the previous assumptions that $\bar{\lambda} \ll a$ and $a \ll d$ are both satisfied, and we shall neglect $\operatorname{Re} h_{+}^{(1)}$. It vanishes exactly if there is no $z$-component to the expectation value of the spin.

\section{Second Order}

There is a restriction on the form of a distortion of the distribution functions $f_{ \pm 1 / 2}$ that is of second order in $\gamma$ (and therefore also of second order in the direction vector $\mathbf{u}$ ), which arises from the requirement that it not alter the values of the particle number density, $n$, and the expectation value of the $z$-component of spin, $\left\langle\sigma_{z}\right\rangle$, both of which are fixed by the terms of zero order. Since these quantities are obtained from Eqs.(15) and (16), the integral over all momenta of the distortions must vanish. The only allowed form is

$$
f_{ \pm 1 / 2}^{(2)}(\mathbf{p})=n_{ \pm 1 / 2} B^{(M)}(p)\left[\mathbf{u} \cdot \mathbf{u}-2 \beta^{(M)}(\mathbf{u} \cdot \mathbf{p})^{2}\right] h_{ \pm 1 / 2}^{(2)}
$$

Indeed, such a form arises naturally from a second application of the operator $\mathbf{u} \cdot \nabla_{p}$ from Eq.(24) on $f_{ \pm 1 / 2}^{(0)}$. For $g_{+}^{(2)}$ we write

$$
g_{+}^{(2)}=n_{+} B^{(M)}(p)(\mathbf{u} \cdot \mathbf{p})^{2} h_{+}^{(2)}(t),
$$

which arises similarly from a second application of $\mathbf{u} \cdot \mathbf{p}$ from Eq. 26) on $g_{+}^{(0)}$.

Now pick out of Eqs.(24)-(26) for the time derivatives of the distribution functions the terms of second order in $\gamma$. When the equations for $\partial f_{ \pm 1 / 2}^{(2)} / \partial t$ are integrated over all $\mathbf{p}$ they become $0=0$. The equation for the time derivative of $g_{+}^{(2)}$ becomes

$$
n_{+} B^{(M)}(p)(\mathbf{u} \cdot \mathbf{p})^{2} \frac{d h_{+}^{(2)}}{d t}=i \frac{1}{M} n_{+} B^{(M)}(p)(\mathbf{u} \cdot \mathbf{p})^{2} F(t) h_{+}^{(1)}+\bar{S}_{+}^{V}(\mathbf{p})
$$


Integrating this equation over all $\mathbf{p}$ and changing the variables of integration in the collision term to $(\mathbf{P}, \mathbf{k})$ that term can be shown to contribute nothing; hence

$$
h_{+}^{(2)}(t)=i \frac{1}{M} \int_{0}^{t} d t F(t) h_{+}^{(1)}(t)
$$

Inserting the expression for $\operatorname{Im} h_{+}^{(1)}$ from Eq.(50) gives

$$
h_{+}^{(2)}(t)=-\frac{1}{M^{2} \alpha} \int_{0}^{t} d t F^{2}(t) .
$$

\section{Time Dependence of $\langle\sigma\rangle$}

We now gather together all the contributions to $\langle\boldsymbol{\sigma}\rangle$, the expectation value of the particle's spin, up to second order in the strength of the gradient of the magnetic field, by inserting into equations $(\sqrt{16})$ and $(\sqrt{17})$ the results from (20), (27) $-(31),(52)$ and (55).

$$
<\sigma_{z}>=<\sigma_{z}>(t=0)
$$

and

$$
\begin{gathered}
<\sigma_{+}>=<\sigma_{+}>(t=0) \cdot \exp \left[-i \gamma\left(B_{0} t+\mathbf{u} \cdot \mathbf{x} F(t)\right)\right] \\
\cdot\left[1-\gamma^{2} D \int_{0}^{t} d t F^{2}(t)+O\left(\gamma^{4}\right)\right]
\end{gathered}
$$

where we have made use of the expression for the diffusion constant $D$ from Eq.(46). Eq.(57) represents the rate of decrease of the expectation value of the transverse component of the particle's spin to second order in the strength of the gradient of the magnetic field.

How does this solution for $\langle\boldsymbol{\sigma}\rangle$ compare with the procedure followed in references [1]-[四] mentioned at the beginning of the paper? This consists of adding a classical diffusion term to the equation for the time rate of change of the magnetization, i.e.,

$$
\frac{\partial \mathbf{M}}{\partial t}=\gamma(\mathbf{M} \times \mathbf{B})+D \nabla^{2} \mathbf{M}
$$

Recall that $\mathbf{M}$ is given by $\langle\boldsymbol{\sigma}>$ multiplied by the magnetic moment of a particle, $\gamma \hbar / 2$, and the number of particles per unit volume. With $\mathbf{B}$ given in Eq.(2) and the initial value of $\mathbf{M}$ independent of postion, the exact solution of Eq.(58) is that $M_{z}$ remains at its initial value; and $M_{+}$is given by

$$
M_{+}(\mathbf{x}, t)=M_{+}(t=0) \exp \left[-i \gamma\left(B_{0} t+\mathbf{u} \cdot \mathbf{x} F(t)\right)\right] \exp \left[-\gamma^{2} D \int_{0}^{t} F^{2}(t) d t\right] .
$$

The final exponential factor is found in references [4] and [12]. If that exponential is expanded for small argument, to second order in $\gamma$ it gives the corresponding term from Eq.(57).

It was stated in the Introduction that the rate of decrease of the transverse component of magnetization is affected by both thermal motion and the interaction of a particle with its environment. By turning off the 2-body potential in Eq.(26), thereby setting the 
collision terms $S^{V}$ to zero, the rate due just to the random velocity distribution can be found, and the exact result is

$$
M_{+}(\mathbf{x}, t)=M_{+}(t=0) \exp \left[-i \gamma\left(B_{0} t+\mathbf{u} \cdot \mathbf{x} F(t)\right)\right] \exp \left[-\gamma^{2} \frac{k T}{M} \underset{\text { (no collisions) }}{\left.\left(\int_{0}^{t} F(t) d t\right)^{2}\right]}\right.
$$

Using expression (46) for the diffusion constant shows that the ratio of the arguments of the exponentials in Eqs. (60) and (59) is qualitatively given by $\alpha T$ where $\alpha^{-1}$ is the mean free time between collisions and $T$ is the duration of the gradient. For the conditions of the experiment in reference [12] $\alpha T \approx 10^{7}$. It is not surprising that the transverse component of magnetization decreases more rapidly without collisions because the particles sample a much larger range of magnetic field strengths in a given time than they would if experiencing collisions, and consequently their precession rates cover a larger range.

For the conditions of the experiment in reference [12] the quantity $\gamma^{2} \int_{0}^{T} F^{2}(t) d t$ has the value $0.032 \mathrm{sec} / \mathrm{mm}^{2}$, where $T$ is the time at which the gradient is turned off. Using the experimental values [15] of the ratio $\left|M_{+}(T) / M_{+}(0)\right|$ of the magnitudes of the transverse magnetization (at various points along the length of the cylinder) after the gradient is turned off to its initial value, and comparing with Eq.(59), the value of the ${ }^{3} \mathrm{He}$ self diffusion coefficient is found to be $D=15.9 \mathrm{~mm}^{2} / \mathrm{sec}$ [15]. The discrepancy with the values shown in Fig.5a of reference [12] is due to a numerical error in that reference [15]. Although the statistical error in the value of $D$ is small, only $0.4 \mathrm{~mm}^{2} / \mathrm{sec}$, there is a much larger systematic error of almost 25 percent because the cell was prepared initially at much higher than room temperature 15].

We also evaluated $D$ from its relation to $\alpha$, Eq.(46), using the estimate given in (47) based on a hard sphere potential with radius $a=2.4 \AA$, and find $D=23.1 \mathrm{~mm}^{2} / \mathrm{sec}$. This falls between the results obtained in previous experiments [19] and [20], after correcting for pressure and isotopic mass dependence, and that reported in [12, 15].

\section{Summary}

The system under consideration consists of spin- $1 / 2$ particles in a magnetic field with a time-varying gradient to its strength, and with its initial magnetization, taken to be spatially uniform, having components both transverse to and along the field direction. We now summarize the line of reasoning that led to the expression (57) for the time development of the expectation value of the transverse component of spin, $\left\langle\sigma_{+}\right\rangle$, starting with Eq.(3) for the time derivative of $\rho$, the 1-body density matrix. It is the off-diagonal elements of $\rho$ in the 2-dimensional spin space, or equivalently the Wigner distribution function $f_{+}$, that determine $<\sigma_{+}>$.

A number of parameters play a role in this development and certain inequalities among them were assumed, in succession. As each of these relations arose we compared the relevant ratios with their values in the experiment reported by Schmidt et al. [12], expressed in a way that allows quick comparison with any other set of experimental parameters. For a low density system of spin $1 / 2$ particles, in the equation for the 2-body density matrix 
we neglected 3-body collisions, and this requires that the mean spacing between particles, $d$, be large compare to the range of the force, $a$. We also assumed that prior to a collision the particles are uncorrelated, so that the 2-body density matrix is the product of the respective 1-body density matrices. Quantum scattering theory is then used to find the effect of a collision.

After expressing the 1-body density matrix elements in terms of Wigner distribution functions, $f$, which form a $2 \times 2$ matrix in spin space, a set of coupled nonlinear integropartial differential equations $(10)$ for the $f$ 's is obtained. On the right hand side are three contributions to their time rate of change: $S^{K}$ arising from the kinetic energy; $S^{\gamma}$ from the magnetic energy; and $S^{V}$ from the 2-body potential energy terms in the Hamiltonian. The first two terms are simple to write down and are given in Eqs.(11) -(14); the expressions for the collision terms $S^{V}$ are obtained in the Appendix, Eqs.(A-17) and (A-18). Two approximations were made to obtain those collision terms. The first is to assume that the 2-body potential is purely central, and this is satisfied extremely well for ${ }^{3} \mathrm{He}$ atoms. The second assumes that the gradient of the magnetic field strength is sufficiently small; this arises from the spatial dependence of the phase factor that governs the Larmor precession, Eq.(20), where $F(t)$ is the time integral of field gradient. We expected and verified that this is the only spatial dependence of the distribution functions. The product $\gamma F(t)$ plays the role of a wave number, and leads to the off diagonal element (in spin space) of the density matrix being non-local in momentum space by the amount $\gamma \hbar F(t)$; see Eq.(22). This nonlocality shifts the arguments of $g_{+}$and the $t$-matrix elements in the collision terms by amounts of the same order, and even leads to non-energy-conserving matrix elements. If these shifts were sizable they would represent important quantum mechanical effects. The magnitude of the effect of the shift on $g_{+}$depends on the ratio of $\gamma \hbar F(t)$ to the mean thermal momenta, and in Eq.(23) this is shown to be very small for the conditions of the experiment in reference [12]. Using a hard sphere potential as a guide, this same small ratio makes the effect of the shift in the arguments of the $t$-matrix elements negligible as well.

To solve the resulting equations (24) - (26) we expanded the distribution functions in a perturbation series to second order in the strength of the field gradient, $G(t)$, using the gyromagnetic ratio $\gamma$ as the expansion coefficient since it multiplies $G$; this makes the equations linear in each order. The only distortions away from Maxwell-Boltzmann distributions that were used are of the form $\mathbf{u} \cdot \mathbf{p}$ in first order and $\mathbf{u} \cdot \mathbf{u}$ and $(\mathbf{u} \cdot \mathbf{p})^{2}$ in second order, where $\mathbf{u}$ is the direction of the gradient. Omitting additional dependence on $p^{2}$ is equivalent to keeping only the leading term in an expansion in Sonine polynomials [13].

To first order in $\gamma$ the equations (A-24) - (A-26) were written still containing the effect of antisymmetization of the wave functions on the collision terms; these are the terms containing $\epsilon$ and they involve, through the quantities $I_{I}$ and $I_{\pi}$ defined in (A-23), not just the absolute square of the $t$-matrix elements but also their real and imaginary parts. For a hard sphere potential in the short wavelength limit, however, the exchange terms are smaller than the direct terms by $O(\bar{\lambda} / a)^{2}$, where $a$ is the hard sphere radius, and we expect this to be true for a more general potential with $a$ the range of the force. But note from Eq.(38) that for ${ }^{3} \mathrm{He}$ at room temperature the ratio $a / \bar{\lambda}$ is only 12.5 with $a=2.4 \AA$. 
At a significantly lower temperature corrections due to the exchange terms would become important; they were neglected in the remainder of the paper. The direct terms, on the other hand, manifest themselves through the single quantity $I_{U}$, which is the mean of the product of the cube of the momentum with the transport cross section $\sigma_{U}$, averaged over a Maxwell-Boltzmann distribution for a particle having the reduced mass.

Provided the field gradient $G(t)$ varies on a time scale much longer than the mean free time between collisions, $\alpha^{-1}$, the solution for the first order distortion of the transverse Wigner distribution function is given in Eqs.(49) and (50). $\alpha$ is simply related to the self diffusion constant $D$ for ${ }^{3} \mathrm{He}$, which can be more readily realized in a different experiment with no field gradient but rather a spatial gradient to the $z$-component of magnetization; this relation is given in Eq. (46). The solution for the second order contribution to the transverse distribution function follows readily from the first order term, and is given in Eq.(55).

If all the restrictions mentioned above are satisfied, then the expression for the rate of decrease of the transverse component of spin, to second order in the strength of the gradient, is given in Eq.(57). Comparison with the classical treatment described in the Introduction, the exact solution for which is given in Eq.(59), shows agreement to second order in the strength of the gradient. It was also shown that the rate would be much greater if there were no collisions, because the particles would sample a much larger range of magnetic field strengths in a given time and consequently their precession rates would also.

It is not possible to make a good comparison between the magnitude of the ${ }^{3} \mathrm{He}$ self diffusion coefficient estimated from a hard sphere potential using Eqs. (46) and (47), and the experimental result in [12] because the temperature varied over a large range [15]. The theoretical estimate of $D=23.1 \mathrm{~mm}^{2} / \mathrm{sec}$ falls between the values obtained in that experiment and that obtained in previous experiments [19, 20].

\section{Acknowledgments}

I am indebted to David Schmidt for introducing me to this subject and for many informative discussions, and for valuable suggestions regarding the manuscript. I also want to thank Emil Motolla and Wojciech Zurek for helpful conversations.

\section{A Appendix}

Here we work out the expressions for the collision terms $S^{V}$. After substituting Eq.(司) for $\rho^{(2)}(1,2)$ in Eq.(18) the elastic scattering $t$-matrix is introduced via the relations [11, 10] $\Omega^{(+)}=\left(I+G^{(+)} t\right)$ and $V \Omega^{(+)}=t$, where $G^{(+)}$is the Green's function. Since the potential conserves the total momentum and the $t$-matrix elements depend only on the relative momenta of the two particles, they can be written

$$
\begin{gathered}
<\mathbf{p}_{A}, m_{A} ; \mathbf{p}_{B}, m_{B}|t| \mathbf{p}_{A}^{\prime}, m_{A}^{\prime} ; \mathbf{p}_{B}^{\prime}, m_{B}^{\prime}>=\delta_{m_{A} m_{A}^{\prime}} \delta_{m_{B} m_{B}^{\prime}} \\
\cdot(2 \pi \hbar)^{3} \delta^{(3)}\left(\mathbf{p}_{A}+\mathbf{p}_{B}-\left(\mathbf{p}^{\prime}{ }_{A}+\mathbf{p}_{B}^{\prime}\right)\right) t\left(\mathbf{k}_{A B}, \mathbf{k}^{\prime}{ }_{A B}\right)
\end{gathered}
$$


where $\mathbf{k}_{A B}=\left(\mathbf{p}_{A}-\mathbf{p}_{B}\right) / 2$. The corresponding equation for the matrix element of $\Omega^{(+)}$is

$$
\begin{gathered}
<\mathbf{p}_{A}, m_{A} ; \mathbf{p}_{B}, m_{B}\left|\Omega^{(+)}\right| \mathbf{p}_{A}^{\prime}, m_{A}^{\prime} ; \mathbf{p}_{B}^{\prime}, m_{B}^{\prime}>=\delta_{m_{A} m_{A}^{\prime}} \delta_{m_{B} m_{B}^{\prime}} \\
\cdot(2 \pi \hbar)^{3} \delta^{(3)}\left(\mathbf{p}_{A}+\mathbf{p}_{B}-\left(\mathbf{p}^{\prime}{ }_{A}+\mathbf{p}_{B}^{\prime}\right)\right) \Omega^{(+)}\left(\mathbf{k}_{A B}, \mathbf{k}_{A B}^{\prime}\right)
\end{gathered}
$$

where

$$
\Omega^{(+)}\left(\mathbf{k}_{A B}, \mathbf{k}_{A B}^{\prime}\right)=\left[(2 \pi \hbar)^{3} \delta^{(3)}\left(\mathbf{p}_{A}-\mathbf{p}_{A}^{\prime}\right)+t\left(\mathbf{k}_{A B}, \mathbf{k}_{A B}^{\prime}\right) /\left(E_{A B}^{\prime}+i \tau-E_{A B}\right)\right]
$$

with $E_{A B}$ the sum of the 1-body energies of particles A and B. (It is understood that the limit $\tau \rightarrow 0$ is to be taken.) Since the magnetic energy term in $H^{(1)}$ is the same in the primed and unprimed states only the kinetic energy contributes to the denominator in Eq. (A-3). Hence $E_{A B}$ can be taken to be $k_{A B}^{2} / 2 \mu$ where $\mu$, the reduced mass, is $M / 2$.

As described in the text we are assuming that the magnitude of $\gamma \hbar F(t)$ is sufficiently small that the shift in the argument of the $\delta$-function in Eq.(22) can be neglected. Hence we shall write

$$
<\mathbf{p}, m|\rho| \mathbf{p}^{\prime}, m^{\prime}>=(2 \pi \hbar)^{3} \delta^{(3)}\left(\mathbf{p}-\mathbf{p}^{\prime}\right) \rho_{m m^{\prime}}(\mathbf{p})
$$

where

$$
\rho_{m m}(\mathbf{p})=(2 \pi \hbar)^{3} f_{m}(\mathbf{p})
$$

and

$$
\rho_{-1 / 2} 1 / 2(\mathbf{p})=(2 \pi \hbar)^{3} g_{+}(\mathbf{p})
$$

When two sets of intermediate states are introduced into Eq.(18) two of the integrations can be carried out immediately by making use of Eqs.(A-1), (A-2), and (A-4), giving

$$
\begin{aligned}
& S_{m m^{\prime}}(\mathbf{x}, \mathbf{p})=-\frac{i}{\hbar} \frac{1}{(2 \pi \hbar)^{3}} \sum_{m_{2}} \int \frac{d^{3} p_{2}}{(2 \pi \hbar)^{3}} \frac{d^{3} p^{\prime}}{(2 \pi \hbar)^{3}} \frac{d^{3} p_{2}^{\prime}}{(2 \pi \hbar)^{3}} \\
& \cdot(2 \pi \hbar)^{3} \delta^{(3)}\left(\mathbf{p}+\mathbf{p}_{\mathbf{2}}-\left(\mathbf{p}^{\prime}+\mathbf{p}_{\mathbf{2}}^{\prime}\right)\right) \\
& \quad\left\{\left[t\left(\mathbf{k}, \mathbf{k}^{\prime}\right) \Omega^{(+) *}\left(\mathbf{k}, \mathbf{k}^{\prime}\right)-\Omega^{(+)}\left(\mathbf{k}, \mathbf{k}^{\prime}\right) t^{*}\left(\mathbf{k}, \mathbf{k}^{\prime}\right)\right] \rho_{m m^{\prime}}\left(\mathbf{p}^{\prime}\right) \rho_{m_{2} m_{2}}\left(\mathbf{p}_{2}^{\prime}\right)\right. \\
&\left.+\epsilon\left[t\left(-\mathbf{k}, \mathbf{k}^{\prime}\right) \Omega^{(+) *}\left(\mathbf{k}, \mathbf{k}^{\prime}\right)-\Omega^{(+)}\left(-\mathbf{k}, \mathbf{k}^{\prime}\right) t^{*}\left(\mathbf{k}, \mathbf{k}^{\prime}\right)\right] \rho_{m_{2} m^{\prime}}\left(\mathbf{p}^{\prime}\right) \rho_{m m_{2}}\left(\mathbf{p}_{2}^{\prime}\right)\right\}
\end{aligned}
$$

After substituting for $\Omega^{(+)}$from Eq. A-3), for the terms linear in the $t$-matrix elements the integrations over the primed variables can be carried out, and for the terms bilinear in $t$ the energy denominators can be combined, giving

$$
\begin{aligned}
& S_{m m^{\prime}}^{V}(\mathbf{x}, \mathbf{p})=\frac{-i}{\hbar}\left\{\frac { 1 } { ( 2 \pi \hbar ) ^ { 3 } } \sum _ { m _ { 2 } } \int \frac { d ^ { 3 } p _ { 2 } } { ( 2 \pi \hbar ) ^ { 3 } } \left(\left[t(\mathbf{k}, \mathbf{k})-t^{*}(\mathbf{k}, \mathbf{k})\right] \rho_{m m^{\prime}}(\mathbf{p}) \rho_{m_{2} m_{2}}\left(\mathbf{p}_{\mathbf{2}}\right)\right.\right. \\
& \left.+\epsilon\left[t(-\mathbf{k}, \mathbf{k}) \rho_{m_{2} m^{\prime}}(\mathbf{p}) \rho_{m m_{2}}\left(\mathbf{p}_{\mathbf{2}}\right)-t^{*}(-\mathbf{k}, \mathbf{k}) \rho_{m_{2} m^{\prime}}\left(\mathbf{p}_{\mathbf{2}}\right) \rho_{m m_{2}}(\mathbf{p})\right]\right) \\
& \quad+\frac{1}{(2 \pi \hbar)^{3}} \sum_{m_{2}} \int \frac{d^{3} p_{2}}{(2 \pi \hbar)^{3}} \frac{d^{3} p^{\prime}}{(2 \pi \hbar)^{3}} \frac{d^{3} p_{2}^{\prime}}{(2 \pi \hbar)^{3}} \\
& \cdot(2 \pi \hbar)^{3} \delta^{(3)}\left(\mathbf{p}+\mathbf{p}_{\mathbf{2}}-\left(\mathbf{p}^{\prime}+\mathbf{p}_{\mathbf{2}}^{\prime}\right)\right) 2 \pi i \delta\left(E^{\prime}-E\right) \\
& \left.\cdot\left[\left|t\left(\mathbf{k}, \mathbf{k}^{\prime}\right)\right|^{2} \rho_{m m^{\prime}}\left(\mathbf{p}^{\prime}\right) \rho_{m_{2} m_{2}}\left(\mathbf{p}_{\mathbf{2}}^{\prime}\right)+\epsilon t\left(-\mathbf{k}, \mathbf{k}^{\prime}\right) t^{*}\left(\mathbf{k}, \mathbf{k}^{\prime}\right) \rho_{m_{2} m^{\prime}}\left(\mathbf{p}^{\prime}\right) \rho_{m m_{2}}\left(\mathbf{p}_{\mathbf{2}}^{\prime}\right)\right]\right\}
\end{aligned}
$$


This establishes the fact that only energy conserving matrix elements contribute to the collision terms, so $t\left(\mathbf{k}, \mathbf{k}^{\prime}\right)$ can be written as $t(k, \theta)$.

Note that any integral of the form

$$
\begin{aligned}
& I\left(\mathbf{p}, \mathbf{p}_{2}\right)=\int \frac{d^{3} p^{\prime}}{(2 \pi \hbar)^{3}} \frac{d^{3} p_{2}^{\prime}}{(2 \pi \hbar)^{3}} \\
& \quad \cdot(2 \pi \hbar)^{3} \delta^{(3)}\left(\mathbf{p}+\mathbf{p}_{\mathbf{2}}-\left(\mathbf{p}^{\prime}+\mathbf{p}_{\mathbf{2}}^{\prime}\right)\right) 2 \pi \delta\left(E^{\prime}-E\right) F\left(\mathbf{p}, \mathbf{p}_{\mathbf{2}}, \mathbf{p}^{\prime}, \mathbf{p}_{\mathbf{2}}^{\prime}\right)
\end{aligned}
$$

can be rewritten by changing the variables of integration to $\left(\mathbf{P}^{\prime}, \mathbf{k}^{\prime}\right)$, where $\mathbf{P}^{\prime}=\mathbf{p}^{\prime}+\mathbf{p}_{\mathbf{2}}^{\prime}$ and $\mathbf{k}^{\prime}=\left(\mathbf{p}^{\prime}-\mathbf{p}_{\mathbf{2}}^{\prime}\right) / \mathbf{2}$. After carrying out the integrations over $\mathbf{P}^{\prime}$ and the magnitude of $\mathbf{k}^{\prime}$, using $E^{\prime}-E=\left(k^{2}-k^{2}\right) / 2 \mu, I$ becomes

$$
I\left(\mathbf{p}, \mathbf{p}_{2}\right)=2 \pi \int \frac{\mu k d \Omega^{\prime}}{(2 \pi \hbar)^{3}} F\left(\mathbf{p}, \mathbf{p}_{2}, \Omega^{\prime}\right)
$$

where $\Omega^{\prime}$ stands for two angles specifying the direction of $\mathbf{k}^{\prime}$. The combination $\mu k d \Omega^{\prime} /(2 \pi \hbar)^{3}$ is the density of final states.

Making use of the relation between the differential scattering cross section and the $t$-matrix elements gives

$$
\begin{aligned}
& v \frac{d \sigma_{U}}{d \Omega^{\prime}}=\frac{2 \pi}{\hbar}\left|t\left(\mathbf{k}, \mathbf{k}^{\prime}\right)\right|^{2} \frac{\mu k}{(2 \pi \hbar)^{3}} \\
& v \frac{d \sigma_{A}}{d \Omega^{\prime}}=\frac{2 \pi}{\hbar}\left|\left[t\left(\mathbf{k}, \mathbf{k}^{\prime}\right)+\epsilon t\left(\mathbf{k},-\mathbf{k}^{\prime}\right)\right] / \sqrt{2}\right|^{2} \frac{\mu k}{(2 \pi \hbar)^{3}}
\end{aligned}
$$

and

$$
v \frac{d \sigma_{I}}{d \Omega^{\prime}}=\frac{2 \pi}{\hbar} t\left(\mathbf{k},-\mathbf{k}^{\prime}\right) t^{*}\left(\mathbf{k}, \mathbf{k}^{\prime}\right) \frac{\mu k}{(2 \pi \hbar)^{3}}
$$

where $\sigma_{U}$ and $\sigma_{A}$ are, respectively, the unsymmetrized and antisymmetrized cross sections. $\sigma_{I}$ is not actually a cross section, just a convenient notation for the interference term; $v=k / \mu$ is the relative velocity of the colliding particles.

Further progress is made by making use of rotational invariance of the $t$-matrix, and unitarity of the $S$-matrix, which takes the form

$$
\begin{aligned}
& 2 i \operatorname{Im} t(\mathbf{k}, \mathbf{k})=-i \int \frac{d^{3} p^{\prime}}{(2 \pi \hbar)^{3}} \frac{d^{3} p_{2}^{\prime}}{(2 \pi \hbar)^{3}}\left|t\left(\mathbf{k}, \mathbf{k}^{\prime}\right)\right|^{2} \\
& \cdot(2 \pi \hbar)^{3} \delta^{(3)}\left(\mathbf{p}+\mathbf{p}_{\mathbf{2}}-\left(\mathbf{p}^{\prime}+\mathbf{p}_{\mathbf{2}}^{\prime}\right)\right) 2 \pi \delta\left(E^{\prime}-E\right),
\end{aligned}
$$

and from the expressions above this becomes

$$
2 i \operatorname{Im} t(\mathbf{k}, \mathbf{k})=-i \hbar \int d \Omega^{\prime} v \frac{d \sigma_{U}}{d \Omega^{\prime}}
$$

The corresponding equation for $t(\mathbf{k},-\mathbf{k})$ is

$$
2 i \operatorname{Im} t(\mathbf{k},-\mathbf{k})=-i \hbar \int d \Omega^{\prime} v \frac{d \sigma_{I}}{d \Omega^{\prime}}
$$


In terms of these quantities the separate cases of $S^{V}$ from Eq. A-8) can be obtained by carrying out the summation over $m_{2}$ and rearranging some terms.

$$
\begin{aligned}
& S_{1 / 2}^{V}(\mathbf{p})=\int d^{3} p_{2} d \Omega^{\prime}\left\{\left[f_{1 / 2}\left(\mathbf{p}^{\prime}\right) f_{-1 / 2}\left(\mathbf{p}_{2}^{\prime}\right)-f_{1 / 2}(\mathbf{p}) f_{-1 / 2}\left(\mathbf{p}_{2}\right)\right] v \frac{d \sigma_{U}}{d \Omega^{\prime}}\right. \\
& \left.+\left[f_{1 / 2}\left(\mathbf{p}^{\prime}\right) f_{1 / 2}\left(\mathbf{p}_{2}^{\prime}\right)-f_{1 / 2}(\mathbf{p}) f_{1 / 2}\left(\mathbf{p}_{2}\right)\right] v \frac{d \sigma_{A}}{d \Omega^{\prime}}+\epsilon g_{+}\left(\mathbf{p}^{\prime}\right) g_{+}^{*}\left(\mathbf{p}_{2}^{\prime}\right) v \frac{d \sigma_{I}}{d \Omega^{\prime}}\right\} \\
& \quad \epsilon \frac{i}{\hbar} \int d^{3} p_{2}\left[t(\mathbf{k},-\mathbf{k}) g_{+}(\mathbf{p}) g_{+}^{*}\left(\mathbf{p}_{2}\right)-c . c .\right]
\end{aligned}
$$

The corresponding equation for $S_{-1 / 2}^{V}$ is obtained from (A-17) by making the interchanges $f_{1 / 2} \leftrightarrow f_{-1 / 2}$ and $g_{+} \leftrightarrow g_{+}^{*}$. Finally, the expression for $\bar{S}_{+}^{V}$ becomes

$$
\begin{aligned}
& \bar{S}_{+}^{V}(\mathbf{p})=\exp \left[i \gamma\left(B_{0} t+\mathbf{u} \cdot \mathbf{x} F(t)\right)\right] S_{+}^{V}(\mathbf{x}, \mathbf{p})=\int d^{3} p_{2} d \Omega^{\prime} \\
& \quad\left\{\left(g_{+}\left(\mathbf{p}^{\prime}\right)\left[f_{1 / 2}\left(\mathbf{p}_{\mathbf{2}}^{\prime}\right)+f_{-1 / 2}\left(\mathbf{p}_{\mathbf{2}}^{\prime}\right)\right]-g_{+}(\mathbf{p})\left[f_{1 / 2}\left(\mathbf{p}_{\mathbf{2}}\right)+f_{-1 / 2}\left(\mathbf{p}_{\mathbf{2}}\right)\right]\right) v \frac{d \sigma_{U}}{d \Omega^{\prime}}\right. \\
& \left.\quad+\epsilon\left[g_{+}\left(\mathbf{p}^{\prime}\right) f_{-1 / 2}\left(\mathbf{p}_{\mathbf{2}}^{\prime}\right)+f_{1 / 2}\left(\mathbf{p}^{\prime}\right) g_{+}\left(\mathbf{p}_{\mathbf{2}}^{\prime}\right)\right] v \frac{d \sigma_{I}}{d \Omega^{\prime}}\right\} \\
& \quad-\epsilon \frac{i}{\hbar} \int d^{3} p_{2}\left(t(\mathbf{k},-\mathbf{k})\left[g_{+}(\mathbf{p}) f_{-1 / 2}\left(\mathbf{p}_{2}\right)+f_{1 / 2}(\mathbf{p}) g_{+}\left(\mathbf{p}_{2}\right)\right]\right. \\
& \left.-t^{*}(\mathbf{k},-\mathbf{k})\left[g_{+}\left(\mathbf{p}_{2}\right) f_{-1 / 2}(\mathbf{p})+f_{1 / 2}\left(\mathbf{p}_{2}\right) g_{+}(\mathbf{p})\right]\right)
\end{aligned}
$$

We now extract the collision terms of first order in $\gamma$, to be used in Eqs.(34) - (36), by inserting Eqs.(32) and (33) in the above. It is helpful to express each individual momentum variable in terms of the total and relative momenta $\mathbf{P}, \mathbf{k}$ and $\mathbf{k}^{\prime}$. When this is done the terms in $S_{ \pm 1 / 2}^{V(1)}$ involving $d \sigma_{A} / d \Omega^{\prime}$ vanish by conservation of momentum; and making use of unitarity, Eq. (A-16), the terms involving the real part of $h_{+}^{(1)}$ cancel, leaving

$$
\begin{aligned}
& S_{1 / 2}^{V(1)}(\mathbf{p})=\mathbf{u} \cdot \int d^{3} p_{2} d \Omega^{\prime} B^{(M)}(p) B^{(M)}\left(p_{2}\right)\left\{n_{1 / 2} n_{-1 / 2}\left(\mathbf{p}^{\prime}-\mathbf{p}\right)\left(h_{1 / 2}^{(1)}-h_{-1 / 2}^{(1)}\right) v \frac{d \sigma_{U}}{d \Omega^{\prime}}\right. \\
& \left.\quad+\epsilon\left|n_{+}\right|^{2} 2 i \mathbf{k}^{\prime} \operatorname{Im} h_{+}^{(1)} v \frac{d \sigma_{I}}{d \Omega^{\prime}}\right\} \\
& \quad+\epsilon\left|n_{+}\right|^{2} \frac{4}{\hbar} \operatorname{Im} h_{+}^{(1)} \mathbf{u} \cdot \int d^{3} p_{2} B^{(M)}(p) B^{(M)}\left(p_{2}\right) \mathbf{k R e} t(\mathbf{k},-\mathbf{k}) .
\end{aligned}
$$

Furthermore, $S_{-1 / 2}^{V(1)}(\mathbf{p})=-S_{1 / 2}^{V(1)}(\mathbf{p})$. Following similar reasoning leads to

$$
\begin{aligned}
& \bar{S}_{+}^{V(1)}(\mathbf{p})=\mathbf{u} \cdot n_{+} \int d^{3} p_{2} d \Omega^{\prime} B^{(M)}(p) B^{(M)}\left(p_{2}\right) \\
& \cdot\left\{\left(\mathbf{p}^{\prime}-\mathbf{p}\right)\left[h_{+}^{(1)}\left(n_{1 / 2}+n_{-1 / 2}\right)-\left(n_{1 / 2} h_{1 / 2}^{(1)}+n_{-1 / 2} h_{-1 / 2}^{(1)}\right)\right] v \frac{d \sigma_{U}}{d \Omega^{\prime}}\right. \\
& \left.+\epsilon \mathbf{k}^{\prime}\left[\left(n_{1 / 2} h_{1 / 2}^{(1)}-n_{-1 / 2} h_{-1 / 2}^{(1)}\right)-\left(n_{1 / 2}-n_{-1 / 2}\right) h_{+}^{(1)}\right] v \frac{d \sigma_{I}}{d \Omega^{\prime}}\right\} \\
& -i \epsilon \frac{2}{\hbar} n_{+}\left[\left(n_{1 / 2} h_{1 / 2}^{(1)}-n_{-1 / 2} h_{-1 / 2}^{(1)}\right)-\left(n_{1 / 2}-n_{-1 / 2}\right) h_{+}^{(1)}\right] \\
& \cdot \mathbf{u} \cdot \int d^{3} p_{2} B^{(M)}(p) B^{(M)}\left(p_{2}\right) \mathbf{k R e t}(\mathbf{k},-\mathbf{k})
\end{aligned}
$$


When these expressions for the first order collision terms are inserted into Eqs. (34) (36) we see that every term is of the form $\mathbf{u} \cdot$ some vector, where $\mathbf{u}$ is an arbitrary direction in space; therefore those equations must be valid as vector equations with the $\mathbf{u}$ omitted. A necessary consequence can be obtained by forming $\mathbf{p}$ - those equations and integrating over all p. At the same time we make the following observations about the collision terms. Change the variables of integration from $\left(\mathbf{p}, \mathbf{p}_{\mathbf{2}}\right)$ to $(\mathbf{P}, \mathbf{k})$, where $\mathbf{P}=\mathbf{p}+\mathbf{p}_{\mathbf{2}}$ and $\mathbf{k}=\left(\mathbf{p}-\mathbf{p}_{\mathbf{2}}\right) / 2$, and make use of

$$
B^{(M)}(p) B^{(M)}\left(p_{2}\right)=B^{(2 M)}(P) B^{(\mu)}(k)
$$

which follows from conservation of energy. For each fixed value of $\mathbf{k}$ choose its direction as the polar axis for the integration over the angles of $\mathbf{k}^{\prime}$, so that $d \Omega^{\prime}$ becomes $d \Omega=2 \pi \sin \theta d \theta$ where $\theta$ is the angle between $\mathbf{k}$ and $\mathbf{k}^{\prime}$. We also define the following quantities.

$$
\begin{aligned}
\sigma_{U}(k) & =\int_{0}^{\pi} d \Omega(1-\cos \theta) \frac{d \sigma_{U}}{d \Omega} \\
\sigma_{I}(k) & =\int_{0}^{\pi} d \Omega \cos \theta \frac{d \sigma_{I}}{d \Omega}
\end{aligned}
$$

and

$$
\begin{aligned}
I_{U} & =\int d^{3} k k^{3} B^{(\mu)}(k) \sigma_{U}(k)=<k^{3} \sigma_{U}(k)>^{(\mu)} \\
I_{I} & =\int d^{3} k k^{3} B^{(\mu)}(k) \sigma_{I}(k)=<k^{3} \sigma_{I}(k)>^{(\mu)} \\
I_{\pi} & =\int d^{3} k k^{2} B^{(\mu)}(k) \operatorname{Ret}(k, \theta=\pi)
\end{aligned}
$$

From the definition of $d \sigma_{I} / d \Omega$, Eq. (A-13), it is seen that $\sigma_{I}(k)$ and $I_{I}$ are pure imaginary, whereas $I_{U}$ and $I_{\pi}$ are real. $\sigma_{U}(k)$ is called the transport cross section [13] and $I_{U}$ is the mean value of $k^{3} \sigma_{U}(k)$ averaged over a Maxwell-Boltzmann distribution for a particle of mass $\mu$.

Eqs. (34) - (36) now become

$$
\begin{aligned}
n_{1 / 2} & \frac{d h_{1 / 2}^{(1)}}{d t}=n_{1 / 2} \hbar \beta^{(M)} G(t) \\
& +\frac{4 \beta^{(M)}}{3 M}\left\{n_{1 / 2} n_{-1 / 2}\left[h_{-1 / 2}^{(1)}-h_{1 / 2}^{(1)}\right] I_{U}-2 \epsilon\left|n_{+}\right|^{2} \operatorname{Im} h_{+}^{(1)} \operatorname{Im} I_{I}\right\} \\
& +\epsilon \frac{8 \beta^{(M)}}{3 \hbar}\left|n_{+}\right|^{2} \operatorname{Im} h_{+}^{(1)} I_{\pi}, \\
n_{-1 / 2} & \frac{d h_{-1 / 2}^{(1)}}{d t}=-n_{-1 / 2} \hbar \beta^{(M)} G(t) \\
& -\frac{4 \beta^{(M)}}{3 M}\left\{n_{1 / 2} n_{-1 / 2}\left[h_{-1 / 2}^{(1)}-h_{1 / 2}^{(1)}\right] I_{U}-2 \epsilon\left|n_{+}\right|^{2} \operatorname{Im} h_{+}^{(1)} \operatorname{Im} I_{I}\right\} \\
& -\epsilon \frac{8 \beta^{(M)}}{3 \hbar}\left|n_{+}\right|^{2} \operatorname{Im} h_{+}^{(1)} I_{\pi}
\end{aligned}
$$


and

$$
\begin{aligned}
& \frac{d h_{+}^{(1)}}{d t}=i \frac{1}{M} F(t)+\frac{4 \beta^{(M)}}{3 M}\left\{\left[n_{1 / 2} h_{1 / 2}^{(1)}+n_{-1 / 2} h_{-1 / 2}^{(1)}-\left(n_{1 / 2}+n_{-1 / 2}\right) h_{+}^{(1)}\right] I_{U}\right. \\
& \left.+\epsilon\left[\left(n_{1 / 2} h_{1 / 2}^{(1)}-n_{-1 / 2} h_{-1 / 2}^{(1)}\right)-\left(n_{1 / 2}-n_{-1 / 2}\right) h_{+}^{(1)}\right] I_{I}\right\} \\
& \quad-i \epsilon \frac{4 \beta^{(M)}}{3 \hbar}\left[\left(n_{1 / 2} h_{1 / 2}^{(1)}-n_{-1 / 2} h_{-1 / 2}^{(1)}\right)-\left(n_{1 / 2}-n_{-1 / 2}\right) h_{+}^{(1)}\right] I_{\pi} .
\end{aligned}
$$

These are four coupled ordinary linear differential equations for the four unknown functions $h_{ \pm 1 / 2}^{(1)}$ and the real and imaginary parts of $h_{+}^{(1)}$.

\section{References}

[1] H. Y. Carr and E. M. Purcell, Phys. Rev. 94, 630 (1954).

[2] H. C. Torrey, Phys. Rev. 104, 563 (1956).

[3] A. Abragam, The Principles of Nuclear Magnetism (Oxford University Press, London, 1961).

[4] E. O. Stejskal and J. E. Tanner, J. Chem. Phys. 42, 288 (1965).

[5] A. Sodickson and D. G. Cory, Prog. Nucl. Mag. Res. Spec. 33, 77 (1998).

[6] E. Wigner, Phys. Rev. 40, 749 (1932).

[7] L. Wolfenstein, "Polarization of Fast Nucleons", in Annual Review of Nuclear Science, J. G. Beckerley, M. D. Kamen, and L. I. Schiff, Eds. (Annual Reviews, Inc., Palo Alto, 1956).

[8] H. D. Zeh, "The Meaning of Decoherence", in Decoherence: Theoretical, Experimental, and Conceptual Problems, Ph. Blanchard, D. Giulini, E. Joos, C. Kiefer, and I.-O. Stamatescu, Eds. (Springer, Berlin, 2000).

[9] C. Lhuillier and F. Laloë, J. Physique 43, 197 (1982); J. Physique 43, 225 (1982); C. Lhuillier, J. Physique 44, 1 (1983).

[10] R. F. Snider, J. Chem. Phys. 32, 1051 (1960).

[11] M. Gell-Mann and M. L. Goldberger, Phys. Rev. 91, 398 (1953).

[12] D. M. Schmidt, J. S. George, S. I. Penttila, A. Caprihan, and E. Fukushima, J. Mag. Res. 129, 184 (1997).

[13] E. M. Lifshitz and L. P. Pitaevskii, Physical Kinetics (Pergamon Press, Oxford, 1981).

[14] R. Feltgen, H. Kirst, K. A. Köhler, H. Pauli, and F. Torello, J. Chem. Phys. 76, 2360 (1982). 
[15] D. M. Schmidt, private communication.

[16] P. M. Morse and H. Feshbach, Methods of Theoretical Physics (McGraw-Hill, New York, 1953).

[17] J. D. Jackson, Classical Electrodynamics (John Wiley and Sons, New York, 1975).

[18] H. S. W. Massey and C. B. O. Mohr, Proc. Royal Soc. London CXLI, 434 (1933).

[19] R. Barbé, M. Leduc, and F. Laloë, J.Physique 35, 935 (1974).

[20] J. C. Liner and S. Weissman, J. Chem. Phys. 56, 2288 (1972). 